\title{
AVALIAÇÃO FUNCIONAL DA DISFAGIA DE LACTENTES EM UTI NEONATAL
}

\author{
Maria Izabel Martins Redondo Botelho*, Ariovaldo Armando da Silva \\ Trabalho realizado na Faculdade de Ciências Médicas - Universidade Estadual \\ de Campinas - UNICAMP, Campinas, SP.
}

RESUMO - OBJETIVos. Os objetivos deste trabalho foram: I) detectar os critérios clínicos na avaliação da disfagia em lactentes sindrômicos e patológicos; 2) testar a hipótese de associação entre achados funcionais e/ou videoendoscópicos; 3) mostrar a importância da atuação conjunta entre o fonoaudiólogo e 0 médico otorrinolaringologista na avaliação da disfagia nesses lactentes.

Métodos. A amostra foi composta por 15 lactentes nascidos no Hospital Maternidade de Campinas (SP), para os quais houve solicitação de uma avaliação fonoaudiológica por parte do médico neonatologista responsável. Todos os lactentes foram avaliados uma vez pelo mesmo fonoaudiólogo e pelo mesmo médico otorrinolaringologista. A avaliação funcional da deglutição observou: a) a sensibilidade táctil extra-oral, b) a sensibilidade táctil intra-oral, c) o reflexo nauseoso e d) a movimentação dos bucinadores. A avaliação da deglutição videoendoscópica analisou: a) sensibilidade na cartilagem aritenóidea, b) o choro, c) a aspiração, d) a queda de saturação, e) a penetração. Utilizou-se o teste exato de Fisher para pequenas amostras e cada hipótese foi testada com nível de significância de 0,05 .

REsultados. Observou-se que existe uma relação de dependência estatisticamente significativa entre as variáveis: I) aspiração após fase faríngea e a penetração (alta e baixa) e 2) sensibilidade extra-oral nas bochechas e a sensibilidade na cartilagem aritenóidea.

Conclusóes. Este último resultado mostra a associação entre um achado funcional obtido pelo fonoaudiólogo e um achado videoendoscópico do médico otorrinolaringologista, o que evidencia a importância do trabalho conjunto desses dois profissionais da saúde.

Unitermos: Disfagia. Lactentes. Deglutição. Avaliação fonoaudiológica. Videoendoscopia da deglutição. UTIneonatal.

\section{INTRODUÇÃO}

A deglutição é dividida em três fases principais: a fase oral, a fase faríngea e a fase esofágica. A deglutição faríngea é uma das primeiras respostas motoras na faringe e está presente em torno da $10^{a}$ e I I ${ }^{a}$ semana navida fetal'. A freqüência respiratória e o volume corrente diminuem durante o ato de alimentação, período em que se observa dessaturação transitória da oxiemoglobina ${ }^{2}$. Assim a imaturidade funcional do lactente, particularmente 0 prematuro, éum dos fatores predisponentesà aspiração. Existe uma relação entre penetração laríngea profunda e aspiração ${ }^{3}$.

Na literatura há várias formas de se classificar as disfagias. Considerou-se as seguintes: I) disfagia segundo a fase da deglutição (oral, faríngea e esofágica) e 2) disfagia segundo a fisiopatologia do distúrbio.

No recém-nascido a disfagia oral geralmente apresenta-se como sucção débil, que pode ser primariamente causada por anorma-

Correspondência:

R. Cel. Spínola de Castro, 4365,

Apto. 164 - Bloco A - Centro

15015-500 - S.J Rio Preto - SP lidades estruturais ou funcionais, como a alteração que os recém-nascidos pré-termo apresentam devido à imaturidade no mecanismo de sucção. As anormalidades da função faríngea levam à dificuldade de alimentação caracterizada por engasgo, tosse, náusea, regurgitação, refluxo faringonasal, baixo ganho ponderal, "distress" respiratório e aspiração, que ocorrem mais na ingestão de líquidos ${ }^{4}$. A disfagia funcional aspirativa é o distúrbio de deglutição mais temido, provocado pela aspiração, que éa passagem de material abaixo das pregas vocais ${ }^{5}$, podendo ocorrer nas seguintes situações: antes da fase faríngea, durante a fase faríngea e depois da fase faríngea.

A adequada avaliação da disfagia deveria ser responsabilidade de uma equipe multidisciplinar. Dela participariam otorrinolaringologistas, fonoaudiólogos, nutricionistas, equipe de enfermagem e outros ${ }^{6}$. A avaliação clínica deve informar a etiologia da disfagia, a possível localização topográfica da dificuldade, os aspectos anatômicos e fisiológicos envolvidos, deve estimar o risco de aspiração pulmonar, discutir testes e procedimentos necessários para o diagnóstico e a terapêutica, bem como métodos alternativos de manuseio na alimentação. As principais etiologias relacionadas à disfagia em neonatos e lactente são: I) prematuridade (distúrbio transitório), 2) anomalias da via aérea/digestiva superior, 3) defeitos congênitos da laringe, traquéia e esôfago, 4) defeitos anatômicos adquiridos e 5) defeitos neurológicos ${ }^{7}$.

O fonoaudiólogo é um dos profissionais responsáveis pela avaliação da disfagia, mas a decisão pelo encaminhamento para uma avaliação deste profissional cabe ao médico neonatologista responsável pelo lactentes. Tal avaliação deve ser feita nos seguintes casos clínicos: utilização de sonda gástrica, sucção fraca, quando existir preocupação com aspiração, vômitos, refluxo nasal, refluxo gastroesofágico, prematuridade, paralisia cerebral e outros ${ }^{1,8,9}$. Não existe um protocolo de avaliação universalmente aceito para avaliar a alimentação em neonatos e/ou lactentes'. Não obstante, alguns autores propuseram roteiros e sugestões de avaliações ${ }^{10,11,12}$. No presente trabalho, os itens selecionados para a pesquisa baseiam-se na avaliação das autoras Glass \& Wolf ${ }^{12}$ : sensibilidade ao toque, reflexo nauseoso, movimentação dos bucinadores e avaliação videoendoscópica da deglutição.

Leder ${ }^{13}$ constatou em sua amostra, que $95 \%$ dos pacientes adultos com reflexo 
nauseoso não apresentaram aspiração laríngea. Também não encontrou relação estatisticamente significativa entre a incidência do reflexo nauseoso e a idade, assim como nenhuma relação foi encontrada entre a prevalência da aspiração e o reflexo nauseoso ou movimento velar na fala ${ }^{13}$.

A avaliação instrumental pode ser realizada por meio de videofluoroscopia, cintilografia, ultra-sonografia e videoendoscopia da deglutição, mas somente a ultra-sonografia e a videofluoroscopia são procedimentos rotineiramente usados para avaliar a disfagia na infância!.

Alguns autores descreveram a avaliação fibroscópica da deglutição, desde técnicas do exame, indicações, contra-indicações e riscos, como também forneceram informações sobre a deglutição de pacientes com disfagias de diferentes causas ${ }^{14,15}$. Manrique, Melo \& Bühler ${ }^{16}$ realizaram a videoendoscopia da deglutição em I 34 crianças portadoras de paralisia cerebral, com idades variando de quatro meses a 14 anos. Eles concluíram que o exame propicia uma avaliação anatômica e funcional das estruturas envolvidas no processo da deglutição, permitindo a obtenção de um grande número de informações essenciais para o diagnóstico e conduta nas disfagias.

$O$ oxímetro de pulso deve ser utilizado durante a alimentação oral de todas as crianças com disfagia grave, para monitorar a saturação $0^{17}$. A saturação é considerada normal em $90 \%$.

Os objetivos deste trabalho são:

I) Detectar os critérios clínicos usados pelo médico neonatologista para a indicação de avaliação da disfagia em lactentes sindrômicos e patológicos;

2) Testar a hipótese de que dois achados funcionais (na fase oral) e/ou videoendoscópicos (na fase faríngea) são independentes um ao outro;

3) Mostrar a importância da atuação conjunta entre o fonoaudiólogo e o otorrinolaringologista na avaliação da disfagia em lactentes sindrômicose patológicos.

\section{Métodos}

\section{Amostra}

Aamostra foi composta por 15 lactentes, nascidos no Hospital Maternidade de Campinas, situado no município de Campinas, esta- do de São Paulo. Os lactentes da amostra tiveram idade gestacional entre 28 semanase 39 semanas e sete dias e solicitação de avaliação fonoaudiológica e otorrinolaringológica pelo médico neonatologista responsável. Todos foram avaliados, por esses profissionais, 28 dias após o nascimento, idade que é classificada pela Pediatria como lactente. Os critérios clínicos que motivaram a solicitação da avaliação constavam da ficha de interconsulta. A coleta de dados foi efetuada em um período de cinco meses. Todos os lactentes pertencentes à amostra foram submetidos a avaliações realizadas por um mesmo médico otorrino-laringologista e pelo mesmo fonoaudiólogo.

\section{Metodologia}

A avaliação da disfagia nos lactentes foi dividida em duas etapas: I) avaliação funcional da deglutição (fase oral), realizada pelo fonoaudiólogo, e 2) avaliação da deglutição videoendoscópica (fase faríngea), realizada pelo médico otorrinolaringologista.

\section{Avaliação funcional da deglutição}

A avaliação realizada pelo fonoaudiólogo iniciou-se com o levantamento da história do prontuário médico do lactente. Os lactentes foram avaliados no colo do fonoaudiólogo. Cada lactente foi avaliado uma única vez, e a avaliação durou em torno de dez minutos. Esta avaliação foi realizada minutos antes do horário da alimentação, devido ànecessidade de os lactentes precisarem estar famintos e em estado de alerta, e consistiu em avaliar:

\section{Sensibilidade táctil extra-oral}

A avaliação da sensibilidade táctil extraoral nas bochechas consiste de um toque com o dedo indicador em uma das bochechas, inicialmente, e depois na outra, observandose a presença ou ausência de reação no lactente. A reação é considerada presente quando o lactente vira a boca, o rosto ou a cabeça, assim também como apresenta movimentos labiais em direção ao toque que foi dado. O procedimento é realizado sem luvas.

O procedimento para a avaliação da sensibilidade táctil extra-oral nos lábios ésemelhante ao anterior, com a diferença que o toque é realizado nas comissuras labiais, lábio superior lábio inferior. Areação é considerada presente quando o lactente responde ao toque, com movimento de boca ou cabeça. A reação no lábio superior pode apresentar-se como uma leve extensão de cabeça e, no lábio inferior, com uma leve flexão de cabeça.

\section{Sensibilidade táctil intra-oral}

Aavaliação da sensibilidade táctil intra-oral na papila palatina consiste em um toque com leve pressão do dedo indicador com luvas, observando-se a presença ou ausência de reação no lactente. A reação é considerada presente quando a língua é direcionada ao dedo do examinador.

A avaliação da sensibilidade nas metades direita e esquerda da língua consiste em um toque realizado por deslizamento na lateral direita (ou esquerda) da língua, iniciando-se na parte anterior da mesma e seguindo para a sua parte posterior. A reação é considerada presente quando a língua lateraliza-se em direção ao estímulo dado. O procedimento é realizado com o dedo indicador, com luvas.

\section{Reflexo nauseoso}

Oreflexo nauseosoé testado com o toque do dedo indicador, com luva, nas regiões: anterior, médio-posterior e posterior da língua, seguindo em direção à úvula, parede posterior da faringe e palato mole. A reação esperada é a abertura da boca, a extensão de cabeça, e a execução de caretas. $O$ reflexo nauseoso foi classificado em: anterior, médioposterior, posterior e ausente.

\section{Movimentação dos bucinadores}

Aavaliação consiste em observar a presença ou ausência de movimentação dos bucinadores durante a sucção nutritiva, como leite administrado na mamadeira e volume prescrito pelo médico neonatologista. A reação será considerada presente quando houver formação de sulcos nas bochechas e, juntamente, formação de bolhas de leite dentro da mamadeira. A reação é considerada ausente quando não há formação de sulcos, ou o movimento é tão débil que não consiga retirar o leite da mamadeira.

\section{Queda de saturação}

Para se verificar se há ou não queda de saturação, utiliza-se um aparelho denominado saturímetro ou oxímetro de pulso. É observada durante a alimentação do lactente. O teste é efetuado com o volume de leite receitado pelo médico neonatologista. 


\section{Avaliação da fase faríngea através da videoendoscopia da deglutição}

Os lactentes foram avaliados em estado de alerta e famintos, para que se pudesse alimentá-los durante o exame, que é feito com o paciente sentado ao colo, sendo todo o procedimento gravado em vídeo. Em seguida, foi realizado a videonasofibroscopia flexível. O exame é feito com o nasofibroscópio Machina 3,2 acoplado a uma micro-câmara, conjugado ao sistema de TV e vídeo, sem uso de anestésico e utilizando-se da narina com permeabilidade após algumas gotas de vasoconstrictor tópico. O lactente é colocado em contenção no colo do fonoaudiólogo sentado e com membros fletidos e em linha média. Neste momento observa-se a intensidade de respostas de repulsa à introdução da fibra. Os lactentes foram monitorados pelo oxímetro de pulso, com o carrinho de emergência ao lado. Atentou-se para a presença ou não de saliva em valéculas ou seios piriformes, ou até mesmo no vestíbulo ou regiões glóticas. Após a alimentação aguarda-se para retirar o nasofibroscópio e capta-se as últimas imagens que podem revelar refluxo pós-alimentação. Durante a realização do exame, 0 neonatologista esteve presente, assim como uma enfermeira.

\section{Variáveis analisadas}

- Sensibilidade nas aritenóides: Asensibilidade nas aritenóides é avaliada pelo toque suave da ponta do nasofibroscópio na mucosa que recobre as cartilagens aritenóides e observa-se se a reação é adequada (quando há reação de adução da glote) ou diminuída (quando não há reação eficiente de adução glótica).

- Choro: Ao introduzir o nasofibroscópio, avalia-se, por meio da discriminação auditiva, a qualidade do choro. Categorias avaliadas: forte, fraco, molhado e ausente.

- Aspiração: Observa-se a passagem de alimento pelas pregas vocais, atingindo a traquéia. Momentos avaliados: aspiração antes da fase faríngea e aspiração após a fase faríngea. Entende-se como aspiração antes da fase faríngea quando houver escape de leite antes do disparo da deglutição. Após a fase faríngea, duas situações podem ocorrer: I) o lactente aspira resíduos de leite depositados nos recessos piriformes ou valéculas, 2) após a deglutição, o leite reflui do estômago e é aspirado.

\begin{tabular}{lc}
\hline \multicolumn{2}{c}{ Tabela I - Freqüência relativa dos distúrbios diagnosticados } \\
\hline Distúrbios & $\%$ \\
Gastroenterológico & 7 \\
Estrutural & 33 \\
Neurológico & 53 \\
Pulmonar & 47 \\
\hline
\end{tabular}

Tabela 2 - Freqüência relativa dos critérios clínicos usados pelo neonatologista para indicação de avaliação do diagnóstico da disfagia em lactentes

$\begin{array}{lr}\text { Critérios clínicos } & \% \\ \text { Usodesonda } & 100 \\ \text { Vômito } & 40 \\ \text { Sucção "débil" } & 33 \\ \text { Nãodegluteasaliva } & 20 \\ \text { Quedadesaturação } & 47 \\ \text { Cianoseperi-oral } & 27 \\ \text { Apnéia } & 13 \\ \text { Dispnéia } & 20\end{array}$

- Queda de saturação: Para severificar se há ou não queda de saturação utiliza-se 0 oxímetro de pulso durante toda a avaliação do lactente. O teste é efetuado com o volume de leite receitado pelo médico neonatologista.

- Penetração: A penetração de alimento no espaço supraglótico foi dividida em penetração alta (até a metade da epiglote sem envolver as bandas ventriculares) e penetração baixa (quando resíduos alimentares envolvem as bandas ventriculares, porém sem ultrapassar a glote).

\section{Tratamento estatístico}

Os dados deste estudo apresentam-se sob a forma de freqüências em categorias discretas, o que exige, para a análise estatística, técnicas não-paramétricas. Procurou-se comprovar a idéia de que duas classificações são independentes uma da outra, comparando-se os seguintes pares de variáveis:

- duas avaliações otorrinolaringológicas: aspiração antes da fase faríngea / penetração alta; aspiração antes da fase faríngea/ penetração baixa; aspiração após a fase faríngea / penetração alta; aspiração após a fase faríngea/ penetração baixa; aspiração antes da fase faríngea/choro; aspiração após a fase faríngea/ choro.

- duas avaliações fonoaudiólogas:movimentação dos bucinadores / sensibilidade táctil extra-oral nas bochechas.
- uma avaliação otorrinolaringológica e uma fonoaudiológica: sensibilidade nas aritenóides/ sensibilidade táctil extra-oral nas bochechas; sensibilidade nas aritenóides / sensibilidade táctil extra-oral nos lábios; sensibilidade nas aritenóides/sensibilidade táctil intraoral na papila palatina; aspiração antes da fase faríngea / reflexo nauseoso; aspiração após a fase faríngea/reflexo nauseoso.

O teste aplicado para determinar a significância foi o teste exato de Fisher. Cada hipótese foi testada com nível de significância de 0,05 .

\section{Resultados}

- Avaliação do médico neonatologista: Foi obtida por meio das fichas interconsultas da UTI da Maternidade de Campinas. Os distúrbios e os critérios clínicos utilizados pelo médico neonatologista responsável para solicitar a avaliação otorrinolaringológica e fonoaudiológica em lactentes são apresentados nas Tabelas I e 2, respectivamente. É necessário salientar que na ficha de interconsulta de cada lactente constavam dois ou mais distúrbios, assim como dois ou mais critérios clínicos.

-Avaliação fonoaudiológica: Os resultados da avaliação funcional da deglutição realizada pelo fonoaudiólogo são apresentados na Tabela 3. Dentre os $67 \%$ que apresentaram reflexo nauseoso ao toque do dedo indicador 


\begin{tabular}{|c|c|c|}
\hline \multirow[t]{2}{*}{ Avaliação } & \multicolumn{2}{|c|}{ Presença } \\
\hline & Sim & Não \\
\hline Sensibilidade Táctilextra-oral-lábios & $53 \%$ & $47 \%$ \\
\hline Sensibilidade Táctilextra-oral-bochechas & $33 \%$ & $67 \%$ \\
\hline Sensibilidade Táctil intra-oral-papilapalatina & $87 \%$ & $13 \%$ \\
\hline Sensibilidade Táctil intra-oral-hemilíngua $\mathrm{D} / \mathrm{E}$ & $100 \%$ & $\%$ \\
\hline Reflexonauseoso & $33 \%$ & $67 \%$ \\
\hline Movimento dos bucinadores/sucção nutritiva & $27 \%$ & $73 \%$ \\
\hline Queda desaturação & $53 \%$ & $47 \%$ \\
\hline
\end{tabular}

\begin{tabular}{|c|c|c|}
\hline \multirow[t]{2}{*}{ Avaliação } & \multicolumn{2}{|c|}{ Presença } \\
\hline & Sim & Não \\
\hline Sensibilidadenasaritenóides & $100 \%$ & $0 \%$ \\
\hline Choro quando da introdução do nasofibroscópio & $100 \%$ & $0 \%$ \\
\hline Alteração da aspiração antes da fase faríngea & $13 \%$ & $87 \%$ \\
\hline Alteração da aspiraçãoapósafasefaríngea & $20 \%$ & $80 \%$ \\
\hline Penetraçãoalta dealimentono espaço supraglótico & $33 \%$ & $67 \%$ \\
\hline Penetração baixa de alimento no espaço supraglótico & $33 \%$ & $67 \%$ \\
\hline Quedadesaturação & $47 \%$ & $53 \%$ \\
\hline
\end{tabular}

\begin{tabular}{lccc}
\hline & $\begin{array}{c}\text { Tabela 5 - Distribuição de freqüências de lactentes de acordo com a } \\
\text { aspiração antes da fase faríngea e a penetração alta }\end{array}$ \\
\hline Penetração & \multicolumn{4}{c}{ Aspiração presente } \\
\cline { 2 - 4 } Alta & Sim & Não & Total \\
Sim & 2 & 3 & 5 \\
Não & 0 & 10 & 10 \\
Total & 2 & 13 & 15 \\
\hline$p=0,095$ & &
\end{tabular}

\begin{tabular}{lccc}
\hline & \multicolumn{3}{c}{$\begin{array}{c}\text { Tabela 6 - Distribuição de freqüências de lactentes de acordo com a } \\
\text { aspiração antes da fase faríngea e a penetração baixa }\end{array}$} \\
\hline Penetração & \multicolumn{4}{c}{ Aspiração presente } \\
Baixa & Sim & Não & Total \\
Sim & 2 & 3 & 5 \\
Não & 0 & 10 & 10 \\
Total & 2 & 13 & 15 \\
\hline$p=0,095$ & &
\end{tabular}

em regiões da língua, 13\% foram na região anterior, $27 \%$ na médio-posterior e $27 \%$ na posterior.

- Avaliação otorrinolaringológica: Os resultados da avaliação da fase faríngea, realizada por meio da videoendoscopia da deglutição é apresentada na Tabela 4. Neste trabalho, a sensibilidade nas aritenóides foi classificada em adequada e diminuída. $\mathrm{Na}$ amostra estudada observou-se que $40 \%$ apresentaram sensibilidade adequada. Por outro lado, o choro foi classificado em forte, fraco, molhado e ausente, como forma de reação à introdução do nasofibroscópio. Os resultados: 74\% apresentaram choro fraco, 13\% choro molhado e $13 \%$ choro forte.

- Análise estatística da associação entre avaliações médicas otorrinolaringológicas.

Com base no teste exato de Fisher, conclui-se que os resultados das Tabelas 5, 6, 9 e 10 não são significativos ao nível de 0,05 , enquanto os resultados das Tabelas 7 e 8 são significativos. Portanto, nesse nível de significância, pode-se dizer que: a) a variável aspiração antes da fase faríngea é independente não só da penetração alta como também da penetração baixa; b) a variável aspiração após a fase faríngea é relacionada tanto com a penetração alta como também com a penetração baixa; c) a variável choro (normal - forte e anormal - fraco, molhado, ausente) é independente tanto da variável aspiração antes da fase faríngea quanto da variável aspiração após a fase faríngea.

-Análise estatística da associação entre avaliações fonoaudiológicas.

Com base no teste exato de Fisher, conclui-se que os resultados da Tabela I I não são significativos ao nível de 0,05. Então, nesse nível de significância, pode-se dizer que não existe uma associação entre a sensibilidade táctil extra-oral nas bochechas e a movimentação dos bucinadores / sucção nutritiva.

- Análise estatística da associação entre avaliações médicas otorrinolaringológicas e avaliações fonoaudiológicas.

Ainda utilizando-se o teste exato de Fisher, conclui-se que os resultados da Tabela I 2 são significativos ao nível de 0,05 , enquanto que os resultados das Tabelas 13, 14, 15 e 16 não o são. Portanto, nesse nível de significância, pode-se afirmar que: a) existe uma associação 


\begin{tabular}{lccc}
\hline \multicolumn{4}{c}{$\begin{array}{c}\text { Tabela } 7 \text { - Distribuição de freqüências de lactentes de acordo com a } \\
\text { aspiração após a fase faríngea e a penetração alta }\end{array}$} \\
\hline Penetração & \multicolumn{3}{c}{ Aspiração presente } \\
\cline { 2 - 4 } Alta & Sim & Não & Total \\
Sim & 3 & 2 & 5 \\
Não & 0 & 10 & 10 \\
Total & 3 & 12 & 15 \\
\hline
\end{tabular}

$p=0,022$

\begin{tabular}{lccc}
\hline \multicolumn{5}{c}{$\begin{array}{c}\text { Tabela } 8 \text { - Distribuição de freqüências de lactentes de acordo com a } \\
\text { aspiração após a fase faríngea e a penetração baixa }\end{array}$} \\
\cline { 2 - 4 } Penetração & \multicolumn{4}{c}{ Aspiraçãa presente } \\
Baixa & Sim & Não & Total \\
Sim & 3 & 2 & 5 \\
Não & 0 & 10 & 10 \\
Total & 3 & 12 & 15 \\
\hline
\end{tabular}

$p=0,022$

\begin{tabular}{cccc}
\hline & $\begin{array}{c}\text { Tabela 9 } \\
\text { - Distribuição de freqüências de lactentes de acordo com a } \\
\text { aspiração antes da fase faríngea e o choro }\end{array}$ \\
\cline { 2 - 4 } Choro & \multicolumn{4}{c}{ Aspiração presente } \\
Normal & Sim & Não & Total \\
Anormal & 0 & 2 & 2 \\
Total & 2 & 11 & 13 \\
\hline
\end{tabular}

$p=0,743$

Tabela 10 - Distribuição de freqüências de lactentes de acordo com a aspiração após a fase faríngea e o choro

\begin{tabular}{lccc}
\hline & \multicolumn{3}{c}{ Aspiração presente } \\
\cline { 2 - 4 } Choro & Sim & Não & Total \\
Normal & 0 & 2 & 2 \\
Anormal & 3 & 10 & 13 \\
Total & 3 & 12 & 15 \\
\hline
\end{tabular}

$p=0,629$

Tabela II - Distribuição de freqüências de lactentes de acordo com a sensibilidade táctil extra-oral nas bochechas e a movimentação dos bucinadores/sucção nutritiva

\begin{tabular}{lccc}
\hline Movimentação & \multicolumn{3}{c}{ Sensibilidade nas bochechas } \\
\cline { 2 - 4 } dos bucinadores & Sim & Não & Total \\
Sim & 3 & 1 & 4 \\
Não & 2 & 9 & 11 \\
Total & 5 & 10 & 15 \\
\hline
\end{tabular}

$p=0,077$ entre a sensibilidade nas aritenóides e a sensibilidade táctil extra-oral nas bochechas; $b$ ) as variáveis sensibilidade táctil extra-oral nos lábios e sensibilidade táctil intra-oral na papila palatina não estão associadas com a sensibilidade nas aritenóides; c) a variável reflexo nauseoso é independente tanto da variável aspiração antes da fase faríngea quanto da variável aspiração após a fase faríngea.

\section{Discussão}

Inúmeras vezes encontram-se lactentes que, por diversas causas, apresentam desordem sensitiva e/ou motora do sistema estomatognático e disfagia. Por meio da sistemática descrita, éapresentada uma possibilidade de avaliar-se estes lactentes. O fonoaudiólogo estaria incumbido da avaliação funcional da fase oral, enquanto o médico otorrinolaringologista se incumbiria da fase faríngea. Assim obter-se-iam melhores soluções para o tratamento do lactente com disfagia.

O avanço da assistência à gravidez de risco e o conseqüente aumento de recém-nascidos pré-termo, sindrômicos e patológicos, têm despertado grande interesse no estudo dos distúrbios de deglutição em neonatos e lactentes. Entretanto são poucas as pesquisas interdisciplinares sobre o assunto.

Nesta pesquisa, os resultados indicam que a patologia mais diagnosticada foi neurológica $(53 \%)$ seguida de problemas pulmonares (47\%), estruturais (33\%) e gastroenterológicos (7\%). Por outro lado, os critérios clínicos utilizados pelo neonatologista para indicação do diagnóstico da disfagia apresentaram-se nas seguintes proporções: $100 \%$ dos lactentes usavam sonda, $47 \%$ tiveram queda de saturação durante as mamadas, $40 \%$ apresentaram vômito, sucção débil (33\%), cianose perioral durante as mamadas (27\%), não-deglutição da saliva (20\%), dispnéia durante as mamadas (20\%) e apnéia durante as mamadas (13\%). Estes resultados estão de acordo com a literatura ${ }^{1,8,9}$.

Neste trabalho, a penetração de alimento no espaço supraglótico foi dividida em penetração alta (até a metade da epiglote, sem envolver as bandas ventriculares) e penetração baixa (quando resíduos alimentares envolvem as bandas ventriculares, porém sem ultrapassar a glote). Observou-se que $67 \%$ dos lactentes não apresentaram penetração, tanto 
Tabela 12 - Distribuição de freqüências de lactentes de acordo com a sensibilidade táctil extra-oral nas bochechas e a sensibilidade nas aritenóides

\begin{tabular}{lccc}
\hline Sensibilidade & \multicolumn{3}{c}{ Sensibilidade nas bochechas } \\
\cline { 2 - 4 } Aritenóidea & Sim & Não & Total \\
Adequada & 4 & 2 & 6 \\
Diminuída & 1 & 8 & 9 \\
Total & 5 & 10 & 15 \\
\hline
\end{tabular}

$p=0,047$

Tabela 13 - Distribuição de freqüências de lactentes de acordo com a sensibilidade táctil extra-oral nos lábios e a sensibilidade nas aritenóides

\begin{tabular}{lccc}
\hline Sensibilidade & \multicolumn{3}{c}{ Sensibilidade nos lábios } \\
\cline { 2 - 4 } Aritenóidea & Sim & Não & Total \\
\cline { 2 - 4 } Adequada & 4 & 2 & 6 \\
Diminuída & 4 & 5 & 9 \\
Total & 8 & 7 & 15 \\
\hline
\end{tabular}

\section{$p=0,377$}

Tabela I4 - Distribuição de freqüências de lactentes de acordo com a sensibilidade táctil intra-oral na papila palatina e a sensibilidade nas aritenóides

\begin{tabular}{lccc}
\hline Sensibilidade & \multicolumn{3}{c}{ Sensibilidade na papila } \\
\cline { 2 - 4 } Aritenóidea & Sim & Não & Total \\
\cline { 2 - 4 } Adequada & 4 & 2 & 6 \\
Diminuída & 9 & 0 & 9 \\
Total & 13 & 2 & 15 \\
\hline
\end{tabular}

$p=0,143$

Tabela I5 - Distribuição de freqüências de lactentes de acordo com a aspiração antes da fase faríngea e o reflexo nauseoso

\begin{tabular}{lccc}
\hline Reflexo & \multicolumn{3}{c}{ Aspiração presente } \\
\cline { 2 - 4 } Nauseoso & Sim & Não & Total \\
\cline { 2 - 4 } Presente & 1 & 9 & 10 \\
Ausente & 1 & 4 & 5 \\
Total & 2 & 13 & 15 \\
\hline
\end{tabular}

$p=0,571$

Tabela 16 - Distribuição de frequêencias de lactentes de acordo com a aspiração após a fase faríngea e o reflexo nauseoso

\begin{tabular}{lccc}
\hline Reflexo & \multicolumn{3}{c}{ Aspiração presente } \\
\cline { 2 - 4 } nauseoso & Sim & Não & Total \\
\cline { 2 - 4 } Presente & 1 & 9 & 10 \\
Ausente & 2 & 3 & 5 \\
Total & 3 & 12 & 15 \\
\hline$p=0,2418$ & &
\end{tabular}

alta como baixa. A penetração laríngea do alimento foi classificada aqui como alta ou bai$x a$, pois existe uma relação entre penetração baixa e aspiração ${ }^{3}$. Friedmann \& Frazier $^{3}$ observaram que, das crianças com penetração laríngea profunda, 85\% apresentaram aspiração, o que segundo eles sugere uma forte correlação entre estes dois eventos.

Neste estudo realizou-se uma avaliação videoendoscópica específica da penetração do alimento (leite materno ou leite receitado pelo médico nenonatologista) e da aspiração do mesmo, observando-se se havia passagem de alimento pelas pregas vocais, atingindo a traquéia. Com relação à aspiração, foi dividida em aspiração antes e aspiração após a fase faríngea. Não se encontrou relação estatisticamente significativa entre a presença de aspiração antes da fase faríngea e I) a penetração alta ou 2) a penetração baixa. Por outro lado, o presente estudo encontrou relação significativa tanto da penetração alta quanto da penetração baixa, com a presença de aspiração após a fase faríngea. Este resultado corrobora aquele obtido por Friedmann \& Frazier ${ }^{3}$.

A existência da queda de saturação foi efetuada por meio de um aparelho denominado oxímetro de pulso, utilizado durante a avaliação. Em seu trabalho, Rogers, Arverdson, Msall, Demerath ${ }^{17}$ preconizam o uso do oxímetro de pulso durante a alimentação oral de todas as crianças com disfagia grave e incapacidades múltiplas. Afreqüência respiratória e o volume corrente diminuem durante o ato de alimentação, período em que se observa dessaturação transitória da oxiemoglobina ${ }^{2}$.A imaturidade funcional do lactente, particularmente o prematuro é, portanto, um dos fatores predisponentes importantes para a aspiração. Neste estudo observou-se que, na avaliação da queda de saturação efetuada pelo fonoaudiólogo, $53 \%$ dos lactentes apresentaram queda (47\% não), enquanto na avaliação posterior, efetuada pelo otorrinolaringologista, houve uma inversão das porcentagens: $47 \%$ apresentaram queda de saturação e $53 \%$ não. Importante ressaltar que todos os lactentes apresentaram recuperação espontânea da saturação em ambas avaliações.

O choro foi classificado em forte, fraco, molhado e ausente, como forma de reação ao nasofibroscópio. Os resultados foram: $74 \%$ apresentaram choro fraco, 13\%, choro molhado, e 13\% choro forte. Nenhum 
lactente deixou de chorar. Nenhuma relação foi encontrada entre a prevalência da aspiração antes da fase faríngea (e da mesma forma para a aspiração após a fase faríngea) e o choro dos lactentes, lembrando que, para o teste de significância, utilizou-se da classificação: choro normal (choro forte) e choro anormal (fraco, molhado e ausente).

Avaliou-se, também, a presença ou ausência de sensibilidade extra-oral nos lábios e nas bochechas, por meio de um toque. Observouse que $53 \%$ dos lactentes apresentaram sensibilidade nos lábios, enquanto apenas 33\% tiveram sensibilidade nas bochechas. Neste trabalho, a sensibilidade nas aritenóides foi classificada em adequada e diminuída. $\mathrm{Na}$ amostra estudada, apenas $40 \%$ apresentaram sensibilidade adequada. Não foi encontrada relação significativa entre a sensibilidade táctil extra-oral nos lábios e sensibilidade nas membranas aritenóideas.

Verificou-se a sensibilidade da parte externa das bochechas e testou-se estatisticamente a hipótese de a presença ou ausência dessa sensibilidade ser independente da presença ou ausência de sensibilidade nas membranas aritenóideas. Após análise estatística, concluiu-se pela existência de uma associação entre as duas variáveis. Do ponto de vista anatômico, são duas estruturas distantes uma da outra (uma é oral e outra é faríngea) e parece não haver uma relação direta entre a sensibilidade extra-oral das bochechas e a sensibilidade nas aritenóides. Entretanto, não se pode descartar a existência de explicação fisiológica e neurofisiológica. Nesse estudo, não foi analisado este resultado sob esses dois ângulos. A estatística, por outro lado, alerta para a ausência de uma relação causa-efeito entre as variáveis. Em outras palavras, significa que a associação encontrada pode ser devida a uma outra variável desconhecida, relacionada com elas. Sugere-se, então, estudos posteriores, com uma nova amostra de maior tamanho, assim como a introdução de novas variáveis para outras análises.

Neste estudo, 87\% dos lactentes apresentaram sensibilidade táctil intra-oral nas papilas palatinas e $13 \%$ não apresentaram nenhuma sensibilidade. Neste estudo não foi encontrada relação significativa entre a sensibilidade táctil intra-oral na papila palatina e sensibilidade nas aritenóides.

Neiva" estudou recém-nascidos pré-termo com idade gestacional de 34 a $366 / 7$ semanas, e encontrou $60 \%$ com movimentação adequada nos músculos bucinadores e $40 \%$ com movimentação excessiva. Nenhum recémnascido deixou de apresentar movimento. Ela classifica a presença de movimentação como contração e descontração contínuas das bochechas. Por outro lado, neste trabalho, $73 \%$ não apresentaram movimentação dos bucinadores quando em presença de sucção nutritiva. Isto se deve, talvez, a o fato de as classificações de ausência de movimentação, nos dois trabalhos, diferirem entre si. Na avaliação desta pesquisa, os lactentes que não apresentavam uma formação de sulcos bem definidos nas bochechas foram considerados com ausência de movimentos dos bucinadores. Não foi encontrada relação estatisticamente significativa entre a sensibilidade táctil extraoral nas bochechas e a movimentação dos músculos bucinadores.

Nesta pesquisa observou-se que $27 \%$ dos lactentes apresentaram reflexo nauseoso posterior, $27 \%$, médio posterior, $13 \%$, anterior e $33 \%$ não apresentaram reflexo nauseoso. Não se encontrou relação estatisticamente significativa entre a presença do reflexo nauseoso e aspiração antes da fase faríngea, da mesma forma que não se observou relação entre a aspiração após a fase faríngea. Tal resultado também foi argumentado por Arvedson \& Brodsky' e encontrado por Leder ${ }^{13}$. De acordo com os primeiros autores, não parece haver relação entre a presença ou ausência do reflexo nauseoso, com a habilidade de deglutição. Já Leder ${ }^{13}$ concluiu que a presença do reflexo nauseoso não protege contra a aspiração, e a ausência desse mesmo reflexo não é uma variável confiável quanto à predição da aspiração, pois não encontrou relação estatisticamente significativa entre a incidência do reflexo nauseoso e a idade, assim como nenhuma relação foi encontrada entre a prevalência da aspiração e o reflexo nauseoso ou movimento velar na fala. Constatou, também, em sua amostra, que 95\% dos pacientes adultos com reflexo nauseoso não apresentaram aspiração laríngea.

Dos sujeitos desta pesquisa, $13 \%$ apresentaram aspiração antes da fase faríngea, enquanto $20 \%$ tiveram aspiração após a fase faríngea. Na pesquisa realizada por Manrique,
Melo, \& Bühler ${ }^{16}$, com I 34 crianças portadoras de paralisia cerebral, com idade entre 4 meses e 14 anos, a aspiração traqueal ocorreu em $33,6 \%$ da amostra, na testagem da consistência líquida. No presente estudo, não se levando em consideração a separação do momento em que ocorreu a aspiração, obteve-se um resultado similar de $33 \%$.

Nesta pesquisa verificou-se que todos os lactentes pertencentes à amostra acusaram presença de sensibilidade nas metades direita e esquerda da língua, após o toque iniciado na parte anterior, seguindo para a parte posterior da mesma. Este resultado não indica a inexistência de lactentes que não apresentem tal sensibilidade.

\section{Conclusóes}

Os critérios clínicos em que se baseia um médico neonatologista para solicitar a avaliação do diagnóstico de disfagia, por um fonoaudiólogo e por um otorrinolaringologista são: uso de sonda; queda de saturação nas mamadas; vômito; sucção débil; cianose perioral nas mamadas; não-deglutição da saliva; dispnéia nas mamadas; apnéia nas mamadas.

A análise dos resultados do teste para indicação de não associação entre os pares de avaliações funcionais e videoendoscópicas permitiu as conclusões abaixo:

- há associação significativa entre a aspiração após a fase faríngea e a penetração alta ou a penetração baixa;

- há associação significativa entre a sensibilidade nas aritenóides e a sensibilidade táctil extra-oral nas bochechas.

- a visível necessidade da atuação conjunta entre o médico otorrinolaringologista e 0 fonoaudiólogo, na avaliação e conduta terapêutica da disfagia em lactentes prétermo, sindrômicos e patológicos, não deixando de lado a importância do trabalho do médico neonatologista e da equipe de enfermagem.

\section{SUMMARY}

THE FUnCtional evaluation OF DYSPHAGY IN THE NEONATAL INTENSIVE CARE UNIT.

BACKGROUND. The objectives of this study were: I) to detect clinical criteria on the evaluation of dysphagy in preterm newborns; 
2) to analyze the association between the functional and the videoendoscopy findings and 3) to show the relevance of the joint work of the Speech Therapist and the Otolaryngologist criteria on the evaluation of dysphagy in preterm syndromic and pathologic newborns.

METHODS. The sample comprised fifteen infants born in Maternity Campinas Hospital, Campinas-SP, to whom was requested a Speech Therapist evaluation by the Neonatologist. All the infants were evaluated once by the same Speech Therapist and the Otolaryngologist. The functional evaluation of swallowing considered: a) the extra oral tactile sensibility, b) the intra oral tactile sensibility, c) the nauseous reflex and d) the buccinator movement. The evaluation of the videoendoscopy swallowing analyzed a) the sensibility in the arytenoid cartilage, b) the crying, c) the aspiration, d) desaturation and e) the penetration. The exact test of Fisher was applied for small samples and each hypothesis was tested with significance level of 0.05 .

RESULTS. At the end of the study, a statistically dependence relation between the variants was noticed: I) aspiration after pharyngeal phase and penetration (high and low) and 2) extra oral sensibility on the cheeks and the sensibility on the arytenoid cartilage.

ConcLusion. This last result shows the association between a functional finding obtained by the Speech Therapist and a videoendoscopy finding achieved by the
Otolaryngologist, that shows clearly the relevance of the team work among these two health professionals. [Rev Assoc Med Bras 2003; 49(3): 278-85]

KEY WORDS. Dysphagy. Preterm newborns. Swallowing. Speech Therapist evaluation. Videoendoscopyswallowing. NICU.

\section{REFERÊNCIAS}

I. Arvedson JCA, Brodsky, L. Pediatric swallowing and feeding-assessment and management. San Diego: Singular Publishing; 1993.

2. Rozov T. Processos aspirativos pulmonares. In: Rozov T. Doenças pulmonares em pediatria - diagnóstico e tratamento. São Paulo: Atheneu; 1999. p.347-52.

3. Friedmann B, Frazier JB. Deep laryngeal penetration as a predictor of aspiration. Dysphagia 2000; 15:153-8.

4. Quintella T, Silva AA, Botelho MIMR. Distúrbios da deglutição (e aspiração) na infância. In: Furkim AM, Santini CS. Disfagias orofaríngeas. Carapicuíba: Pró-Fono; 1999. p.61-96.

5. Perlman A, Debrieu KS. Deglution and its disorders. San Diego: Singular Publishing Group; 1997.

6. Xavier C. Trabalho fonoaudiológico com bebês durante a fase hospitalar. In: Limongi SCO. Paralisia cerebral: processo terapêutico em linguagem e cognição. Carapicuíba: PróFono; 2000. p. 75-II8.

7. Tuchman DN, Walter RS. Disorders of feeding and Swallowing in infants and children pathology, diagnosis and treatment. San Diego: Singular Publishing Group Inc; 1994.

8. Mathew OP. Science of bottle feeding. J Pediatr 1991; I| 19:5।|-9.

9. Xavier C. Assistência à alimentação de bebês hospitalizados. In: Bassetto MCA. Neonatologia: um convite à atuação fonoaudiológica. São Paulo: Lovise; 1998. p 255-75.

10. Braun MA, Palmer MM. A pilot study of oralmotor dysfunction in "at-risk" infants. Phys Occup Ther Pediatr 1986; 5: 1 3-25.

I I. Neiva FCB. Análise do padrão de sucção em recém-nascidos de termo e pré-termo com idade gestacional de 34 a 36 6/7 semanas (dissertação). São Paulo: Universidade de São Paulo; 1999.

12. Glass RP, Wolf LS. A global perspective on feeding assessment in the neonatal intensive care unit. Am J Occup Ther 1994; 48:5।4-26.

13. Leder SB. Videofluoroscopic evaluation of aspiration with visual examination of the gag reflex and velar movement. Dysphagia 1997; 12:21-3.

14. Langmore SE, Mcculloch TM. Examination of the pharynx and larynx and endoscopic examination of pharyngeal. In: Perlman AL, Delrieu KS. Deglutition and its disorders. San Diego: Singular Publishing Group; 1997. p. 20I-26.

15. Macedo Filho E. Avaliação endoscópica da deglutição (FEESS) na abordagem da disfagia orofaríngea. In: Macedo Filho E. Disfagia: abordagem multidisciplinar. São Paulo: Frôntis Editorial; 1998. p.69-76.

16. Manrique D, Melo ECM, Bühler RB. Avaliação nasofibrolaringoscópica da deglutição em crianças. Rev Bras Otorrinolaringol 200I; 67:796-80I.

17. Rogers BT, Arverdson J, Msall M, Demerath RR. Hypoxemia during oral feeding of children with severe cerebral palsy. Dev Med Child Neurol 1993; 35:3-9.

Artigo recebido: 26/07/02 Aceito para publicação: 14/03/03 